\title{
Blow-up phenomena and stability of solitary waves for a generalized Dullin-Gottwald-Holm equation
}

\author{
Nurhan Dündar* and Necat Polat
}

"Correspondence:

nurhandundar@hotmail.com

Department of Mathematics, Dicle

University, Diyarbakir, 21280, Turkey

\begin{abstract}
In this work, we consider the Cauchy problem of the generalized Dullin-Gottwald-Holm equation. We establish a blow-up result for the generalized Dullin-Gottwald-Holm equation. In addition to this, we investigate the stability of solitary wave solutions of the equation.
\end{abstract}

Keywords: the generalized Dullin-Gottwald-Holm equation; blow-up; stability; solitary wave solution

\section{Introduction}

The nonlinear evolution equation

$$
y_{t}+c_{0} u_{x}+u y_{x}+2 y u_{x}+\gamma u_{x x x}=0, \quad t>0, x \in R
$$

is, in the dimensionless space-time variable $(x, t)$, a model for unidirectional shallow water waves over a flat bottom. Here, $y=u-\alpha^{2} u_{x x}$ is a momentum variable, the constants $\alpha^{2}$ and $\frac{\gamma}{c_{0}}$ are squares of length scales, and $c_{0}=\sqrt{g h}>0$ (where $c_{0}=2 \omega$ ) is the linear wave speed for undisturbed water at rest at spatial infinity, where $h$ is the mean fluid depth and $g$ is the gravitational constant. Dullin, Gottwald and Holm derived Eq. (1) by using asymptotic expansions directly in the Hamiltonian for Euler's equations in the shallow water regime in [1]. Eq. (1) was shown to be bi-Hamiltonian and to have a Lax pair formulation. The Dullin-Gottwald-Holm equation (we call it DGH equation for short) is an integrable system via the inverse scattering transform (IST) method and contains both the Korteweg-de Vries (KdV) and Camassa-Holm $(\mathrm{CH})$ equations [2] as limiting cases.

Using the notation $y=u-\alpha^{2} u_{x x}$, we can rewrite the initial value problem of Eq. (1) as

$$
\left\{\begin{array}{l}
u_{t}-\alpha^{2} u_{x x t}+2 \omega u_{x}+3 u u_{x}+\gamma u_{x x x} \\
\quad=\alpha^{2}\left(2 u_{x} u_{x x}+u u_{x x x}\right), \quad t>0, x \in R \\
u(0, x)=u_{0}(x), \quad x \in R
\end{array}\right.
$$

Eq. (2) relates two separately integrable soliton equations for water waves. Formally, when $\alpha=0$ and $\gamma \neq 0$, this equation becomes the $\mathrm{KdV}$ equation

$$
u_{t}+2 \omega u_{x}+3 u u_{x}+\gamma u_{x x x}=0
$$


For $\alpha=1$ and $\gamma=0$, Eq. (2) becomes the Camassa-Holm equation

$$
u_{t}-u_{x x t}+2 \omega u_{x}+3 u u_{x}=2 u_{x} u_{x x}+u u_{x x x} .
$$

Recently, the DGH equation has been studied by many authors. Tian et al. [3] studied the well-posedness of the Cauchy problem and the scattering problem for the DGH equation. In [4], Hakkaev proved the orbital stability of the peaked solitary waves for the DGH equation by using the method in [5]. It was shown that the DGH equation has global solutions and blow-up solutions in [6-9].

If the term $3 u u_{x}$ is replaced with $3 u^{m} u_{x}$ in the DGH equation, then it is known as the generalized DGH equation and has the following form:

$$
u_{t}-\alpha^{2} u_{x x t}+2 \omega u_{x}+3 u^{m} u_{x}+\gamma u_{x x x}=\alpha^{2}\left(2 u_{x} u_{x x}+u u_{x x x}\right), \quad m>0 .
$$

Eq. (3) was studied in [10, 11]. In [10], Lu et al. studied the local well-posedness of the Cauchy problem for Eq. (3). In [11], the conservation laws for the generalized DGH equation were derived.

Recently, the local well-posedness problem for the following generalization of the DGH equation:

$$
\left\{\begin{array}{l}
u_{t}-\alpha^{2} u_{x x t}+h(u)_{x}+\gamma u_{x x x}=\alpha^{2}\left(\frac{g^{\prime}(u)}{2} u_{x}^{2}+g(u) u_{x x}\right)_{x}, \quad t>0, x \in R, \\
u(0, x)=u_{0}(x), \quad x \in R,
\end{array}\right.
$$

was studied in [12]. For $h(u)=2 \omega u+\frac{3}{2} u^{2}$ and $g(u)=u$, Eq. (4) becomes DGH Eq. (2).

Eq. (4) can be written as the following Hamiltonian form:

$$
u_{t}+J F^{\prime}(u)=0
$$

where $J=\left(I-\alpha^{2} \partial_{x}^{2}\right)^{-1} \partial_{x}$ is a skew-symmetric operator and

$$
F(u)=\frac{1}{2} \int_{R}\left(2 H(u)+\alpha^{2} g(u) u_{x}^{2}-\gamma u_{x}^{2}\right) d x
$$

where $H^{\prime}(s)=h(s)$. We note that the functional $F(u)$ is formally conserved. Moreover, the other conserved quantity is

$$
E(u)=\frac{1}{2} \int_{R}\left(u^{2}+\alpha^{2} u_{x}^{2}\right) d x .
$$

One of the aims of this paper is to give the precise blow-up scenario and to show that Eq. (4) has blow-up solutions for $g(u)=u$. In addition to this, we investigate the stability of solitary wave solutions of Eq. (4) with $h(u)=2 \omega u+\frac{p+2}{2} u^{p+1}$ and $g(u)=u^{p}$.

The remainder of the paper is organized as follows. In Section 2, we give our basic notation and recall some required results. In Section 3, we investigate blow-up of solutions for Eq. (4). In Section 4, we prove the stability of solitary wave solutions with the help of the orbital stability theory [13]. 


\section{Preliminaries}

Firstly, we start by summarizing some notations. $\Lambda^{s}=\left(1-\partial_{x}^{2}\right)^{s / 2}, s \in R ; H^{s}=H^{s}(R)$ with the norm

$$
\|f\|_{H^{s}}=\|f\|_{s}=\left(\left.\int_{R}\left(1+|\xi|^{2}\right)^{s} \widehat{f}(\xi)\right|^{2} d \xi\right)^{1 / 2}
$$

and $(\cdot, \cdot)$ for its inner product. For the sake of simplicity, we employ the same symbol $c$ for different positive constants.

Some useful lemmas are as follows.

Lemma 2.1 [14] Assume that $s>0$. Then we have

$$
\left\|\left[\Lambda^{s}, g\right] f\right\|_{L^{2}} \leq c\left(\left\|\partial_{x} g\right\|_{L^{\infty}}\left\|\Lambda^{s-1} f\right\|_{L^{2}}+\left\|\Lambda^{s} g\right\|_{L^{2}}\|f\|_{L^{\infty}}\right) .
$$

Here $c$ is a constant depending only on $s$.

Lemma $2.2[15]$ Assume that $F \in C^{m+2}(R, R)$ with $F(0)=0$. Then, for every $\frac{1}{2}<s \leq m$, we have that

$$
\|F(u)\|_{s} \leq \widetilde{F}\left(\|u\|_{L^{\infty}}\right)\|u\|_{s}, \quad u \in H^{s}
$$

where $\widetilde{F}$ is a monotone increasing function depending only on $F$ and $s$.

Lemma 2.3 [14] Assume that $s>0$. Then $H^{s} \cap L^{\infty}$ is an algebra. Moreover,

$$
\|f g\|_{s} \leq c\left(\|f\|_{L^{\infty}}\|g\|_{s}+\|f\|_{s}\|g\|_{L^{\infty}}\right)
$$

where $c$ is a constant depending only on s.

Lemma 2.4 [16] Let $T>0$ and $u \in C^{1}\left([0, T) ; H^{2}\right)$. Then, for every $t \in[0, T)$, there exists at least one pair of points $\xi(t), \zeta(t) \in R$ such that

$$
m(t)=\inf _{x \in R}\left[u_{x}(t, x)\right]=u_{x}(t, \xi(t)), \quad M(t)=\sup _{x \in R}\left[u_{x}(t, x)\right]=u_{x}(t, \zeta(t)),
$$

and $m(t), M(t)$ are absolutely continuous in $(0, T)$. Moreover,

$$
\frac{d m(t)}{d t}=u_{t x}(t, \xi(t)), \quad \frac{d M(t)}{d t}=u_{t x}(t, \zeta(t)), \quad \text { a.e. on }(0, T) .
$$

Theorem 2.1 [12] Assume that $h, g \in C^{m+3}(R, R), m \geq 2$ and $h(0)=g(0)=0$. Given $u_{0} \in H^{s}, \frac{3}{2}<s \leq m$, there exists a maximal $T=T\left(u_{0}\right)>0$ and a unique solution $u$ to Eq. (4) such that

$$
u=u\left(\cdot, u_{0}\right) \in C\left([0, T) ; H^{s}\right) \cap C^{1}\left([0, T) ; H^{s-1}\right) .
$$

Moreover, the solution depends continuously on the initial data, i.e., the mapping

$$
u_{0} \rightarrow u\left(\cdot, u_{0}\right): H^{s} \rightarrow C\left([0, T) ; H^{s}\right) \cap C^{1}\left([0, T) ; H^{s-1}\right)
$$


Lemma 2.5 [12] Let $u(t, x)$ be a solution of Eq. (4). Then the functionals

$$
E(u)=\frac{1}{2} \int_{R}\left(u^{2}+\alpha^{2} u_{x}^{2}\right) d x, \quad F(u)=\frac{1}{2} \int_{R}\left(2 H(u)+\alpha^{2} g(u) u_{x}^{2}-\gamma u_{x}^{2}\right) d x
$$

are constant with respect to $t$, where $H^{\prime}(s)=h(s)$.

\section{Blow-up phenomena}

In this section, we discuss the blow-up phenomena of Eq. (4) with $g(u)=u$. For Eq. (4), which describes shallow water waves, the blow-up occurs only in the form of wavebreaking, i.e. the solution remains bounded but its slope becomes unbounded in finite time.

Set $p(x)=\frac{1}{2 \alpha} e^{-\left|\frac{x}{\alpha}\right|}, x \in R$, then $\left(1-\alpha^{2} \partial_{x}^{2}\right)^{-1} f=p * f$ for all $f \in L^{2}(R)$. Using this identity, we can rewrite Eq. (4) as follows:

$$
\left\{\begin{array}{l}
u_{t}+\left(u-\frac{\gamma}{\alpha^{2}}\right) u_{x}+\partial_{x} p * k(u)=0, \quad t>0, x \in R \\
u(0, x)=u_{0}(x), \quad x \in R
\end{array}\right.
$$

or in the equivalent form:

$$
\left\{\begin{array}{l}
u_{t}+\left(u-\frac{\gamma}{\alpha^{2}}\right) u_{x}=-\partial_{x}\left(1-\alpha^{2} \partial_{x}^{2}\right)^{-1} k(u), \quad t>0, x \in R \\
u(0, x)=u_{0}(x), \quad x \in R
\end{array}\right.
$$

where $k(u)=\left(h(u)+\frac{\alpha^{2}}{2} u_{x}^{2}-\frac{1}{2} u^{2}+\frac{\gamma}{\alpha^{2}} u\right)$.

We now prove the following result.

Theorem 3.1 Let $h \in C^{m+3}(R, R), m \geq 2$, and $u_{0} \in H^{r}, \frac{3}{2}<r \leq m$. If $T$ is the existence time of the corresponding solution of initial data $u_{0}$, then the $H^{r}$-norm of $u(t, x)$ to Eq. (4) (or (6)) blows up on $[0, T)$ if and only if

$$
\varlimsup_{t \rightarrow T}\left\{\|u(t, x)\|_{L^{\infty}}+\left\|u_{x}(t, x)\right\|_{L^{\infty}}\right\}=\infty .
$$

Proof Let $u(t, x)$ be the solution of Eq. (4) with the initial data $u_{0} \in H^{r}, \frac{3}{2}<r \leq m$, which is guaranteed by Theorem 2.1. If

$$
\varlimsup_{t \rightarrow T}\left\{\|u(t, x)\|_{L^{\infty}}+\left\|u_{x}(t, x)\right\|_{L^{\infty}}\right\}=\infty,
$$

by Sobolev's embedding theorem, we obtain that the solution $u(t, x)$ will blow up in finite time.

Next, applying the operator $\Lambda^{r}$ to Eq. (6), multiplying by $\Lambda^{r} u$, and integrating over $R$, we obtain

$$
\frac{d}{d t}(u, u)_{r}=-2\left(u u_{x}, u\right)_{r}+2(f(u), u)_{r} .
$$


Here, $f(u)=-\partial_{x}\left(1-\alpha^{2} \partial_{x}^{2}\right)^{-1}\left(h(u)+\frac{\alpha^{2}}{2} u_{x}^{2}-\frac{1}{2} u^{2}+\frac{\gamma}{\alpha^{2}} u\right)$. Assume that there exists $R>0$ such that

$$
\varlimsup_{t \rightarrow T}\left\{\|u(t, x)\|_{L^{\infty}}+\left\|u_{x}(t, x)\right\|_{L^{\infty}}\right\} \leq R
$$

Similar to [18], using Lemma 2.1 with $s=r$, we get

$$
\left|\left(u u_{x}, u\right)_{r}\right| \leq c\left\|u_{x}\right\|_{L^{\infty}}\|u\|_{r}^{2} \leq c R\|u\|_{r}^{2}
$$

On the other hand, we estimate the second term of the right-hand side of Eq. (7)

$$
\begin{aligned}
(f(u), u)_{r} & =\left(-\partial_{x}\left(1-\alpha^{2} \partial_{x}^{2}\right)^{-1}\left(h(u)+\frac{\alpha^{2}}{2} u_{x}^{2}-\frac{1}{2} u^{2}+\frac{\gamma}{\alpha^{2}} u\right), u\right)_{r} \\
& \leq c\|u\|_{r}\left(\|h(u)\|_{r-1}+\left\|u_{x}^{2}\right\|_{r-1}+\left\|u^{2}\right\|_{r-1}+\|u\|_{r-1}\right) \\
& \leq c\left(\tilde{h}\left(\|u\|_{L^{\infty}}\right)+\left\|u_{x}\right\|_{L^{\infty}}+\|u\|_{\left.L^{\infty}+1\right)\|u\|_{r}^{2}}\right. \\
& \leq c(\tilde{h}(R)+R+1)\|u\|_{r}^{2} .
\end{aligned}
$$

Here, we applied Lemma 2.2 with $F(u)=h(u)$ and $s=r-1$, Lemma 2.3 with $s=r-1$. From (7)-(9), we obtain

$$
\frac{d}{d t}\|u\|_{r}^{2} \leq c(\tilde{h}(R)+R+1)\|u\|_{r}^{2} .
$$

Thus, by Gronwall's inequality, we get

$$
\|u(t)\|_{r}^{2} \leq\left\|u_{0}\right\|_{r}^{2} \exp (\widetilde{h}(R)+R+1) t
$$

This completes the proof of Theorem 3.1.

We have the following blow-up scenario for Eq. (4).

Theorem 3.2 Assume that $h \in C^{m+3}(R, R), m \geq 3$. Given $u_{0} \in H^{r}, 3 \leq r \leq m$, the solution $u=u\left(u_{0}, \cdot\right)$ of Eq. (4) is uniformly bounded. Blow-up in finite time $T<+\infty$ occurs if and only if

$$
\liminf _{t \rightarrow T}\left\{\inf _{x \in R}\left[u_{x}(t, x)\right]\right\}=-\infty
$$

Proof $E(u)=\frac{1}{2} \int_{R}\left(u^{2}+\alpha^{2} u_{x}^{2}\right) d x$ is an invariant for Eq. (4). According to the inequalities

$$
\begin{aligned}
& \int_{R}\left(u^{2}+u_{x}^{2}\right) d x \leq \int_{R}\left(u^{2}+\alpha^{2} u_{x}^{2}\right) d x=2 E(u) \quad(\alpha \geq 1), \\
& \int_{R}\left(u^{2}+u_{x}^{2}\right) d x \leq \frac{1}{\alpha^{2}} \int_{R}\left(u^{2}+\alpha^{2} u_{x}^{2}\right) d x=\frac{2}{\alpha^{2}} E(u) \quad(\alpha \leq 1),
\end{aligned}
$$

the invariance of $E(u)$ ensures that the solution $u$ is uniformly bounded as long as they exist. 
If the slope of the solution $u(t, x)$ becomes unbounded from below in finite time, then by Theorem 2.1 and Sobolev's embedding theorem, we can see that the solution $u(t, x)$ blows up in finite time.

Next, if the slope of the solution is bounded from below in finite time, then we deduce that the solution will not blow up in finite time. Differentiating Eq. (5) with respect to $x$, in view of the identity $\partial_{x}^{2}(p * f)=\frac{1}{\alpha^{2}}(p * f-f)$, we have

$$
\begin{aligned}
u_{t x}= & -\frac{1}{2} u_{x}^{2}-u u_{x x}+\frac{\gamma}{\alpha^{2}} u_{x x}+\frac{1}{\alpha^{2}} h(u)-\frac{1}{2 \alpha^{2}} u^{2}+\frac{\gamma}{\alpha^{4}} u \\
& -\frac{1}{\alpha^{2}} p *\left(h(u)+\frac{\alpha^{2}}{2} u_{x}^{2}-\frac{1}{2} u^{2}+\frac{\gamma}{\alpha^{2}} u\right) .
\end{aligned}
$$

Note that $p *\left(\frac{1}{2} u_{x}^{2}\right) \geq 0$ and

$$
\|u\|_{L^{\infty}}^{2} \leq \frac{1}{2}\|u\|_{1}^{2} \leq \max \left(1, \frac{1}{\alpha^{2}}\right) E\left(u_{0}\right) \leq \max \left(1, \frac{1}{\alpha^{4}}\right)\left\|u_{0}\right\|_{1}^{2} .
$$

By Young's inequality, we get

$$
\begin{aligned}
& \|p * h(u)\|_{L^{\infty}} \leq\|p\|_{L^{1}}\|h(u)\|_{L^{\infty}} \leq\|h(u)\|_{L^{\infty}} \leq \sup _{|v| \leq \max \left(1, \frac{1}{\alpha^{2}}\right)\left\|u_{0}\right\|_{1}}|h(v)|, \\
& \left\|p * u^{2}\right\|_{L^{\infty}} \leq\|p\|_{L^{1}}\left\|u^{2}\right\|_{L^{\infty}} \leq\|u\|_{L^{\infty}}^{2} \leq \max \left(1, \frac{1}{\alpha^{4}}\right)\left\|u_{0}\right\|_{1}^{2}
\end{aligned}
$$

and

$$
\|p * u\|_{L^{\infty}} \leq\|p\|_{L^{1}}\|u\|_{L^{\infty}} \leq\|u\|_{L^{\infty}} \leq \max \left(1, \frac{1}{\alpha^{2}}\right)\left\|u_{0}\right\|_{1} .
$$

Define $M(t)=u_{x}(t, \zeta(t))=\sup _{x \in R}\left[u_{x}(t, x)\right]$. Since $u_{x x}(t, \zeta(t))=0$ for all $t \in[0, T)$, it follows that a.e. on $[0, T)$

$$
\begin{aligned}
M^{\prime}(t)= & -\frac{1}{2} M^{2}(t)+\frac{1}{\alpha^{2}} h(u(t, \zeta(t)))-\frac{1}{2 \alpha^{2}} u^{2}(t, \zeta(t))+\frac{\gamma}{\alpha^{4}} u(t, \zeta(t)) \\
& -\frac{1}{\alpha^{2}} p *\left(h(u)+\frac{\alpha^{2}}{2} u_{x}^{2}-\frac{1}{2} u^{2}+\frac{\gamma}{\alpha^{2}} u\right) .
\end{aligned}
$$

Then

$$
M^{\prime}(t) \leq-\frac{1}{2} M^{2}(t)+A_{0}^{2}
$$

where

$$
A_{0}=\left(\frac{2 \gamma}{\alpha^{4}} \max \left(1, \frac{1}{\alpha^{2}}\right)\left\|u_{0}\right\|_{1}+\frac{1}{2 \alpha^{2}} \max \left(1, \frac{1}{\alpha^{4}}\right)\left\|u_{0}\right\|_{1}^{2}+\frac{2}{\alpha^{2}} G_{0}\right)^{\frac{1}{2}}
$$

by

$$
G_{0}=\sup _{|v| \leq \max \left(1, \frac{1}{\alpha^{2}}\right)\left\|u_{0}\right\|_{1}}|h(v)| .
$$


If $M(t)>\sqrt{2} A_{0}$, then $M^{\prime}(t)<0$ and $M(t)$ is decreasing. Otherwise, $M(t) \leq \sqrt{2} A_{0}$. Thus we get

$$
m(t) \leq M(t) \leq \max \left\{M(0), \sqrt{2} A_{0}\right\}, \quad t \in[0, T) .
$$

By Theorem 3.1 and the above inequality, we have that if the slope of the solution is bounded from below in finite time, then the solution will not blow up in finite time.

Next, we present the following blow-up result.

Theorem 3.3 Assume that $h \in C^{m+3}(R, R), m \geq 3$. Given $\alpha>0, u_{0} \in H^{r}, 3 \leq r \leq m$, assume that we can find $x_{0} \in R$ with

$$
\begin{aligned}
u_{0}^{\prime}\left(x_{0}\right) & <-\left(\frac{4 \gamma}{\alpha^{4}} \max \left(1, \frac{1}{\alpha^{2}}\right)\left\|u_{0}\right\|_{1}+\frac{1}{\alpha^{2}} \max \left(1, \frac{1}{\alpha^{4}}\right)\left\|u_{0}\right\|_{1}^{2}+\frac{4}{\alpha^{2}} G_{0}\right)^{\frac{1}{2}} \\
& =-\sqrt{B_{0}}
\end{aligned}
$$

where

$$
G_{0}=\sup _{|v| \leq \max \left(1, \frac{1}{\alpha^{2}}\right)\left\|u_{0}\right\|_{1}}|h(v)| .
$$

Then the corresponding solution to Eq. (4) for $g(u)=u$ blows up in finite time. Moreover, the maximal time of existence $T$ satisfies the inequality

$$
T \leq 2 c\left(c^{2}-B_{0}\right)^{-1}, \quad \text { where } c=-u_{0}^{\prime}\left(x_{0}\right)
$$

Proof Now define $m(t)=\inf _{x \in R}\left[u_{x}(t, x)\right]=u_{x}(t, \xi(t))$ by Lemma 2.4, and let $\xi(t) \in R$ be a point where this infimum is attained. From Eq. (10), we have

$$
\begin{aligned}
m^{\prime}(t)= & -\frac{1}{2} m^{2}(t)+\frac{1}{\alpha^{2}} h(u(t, \xi(t)))-\frac{1}{2 \alpha^{2}} u^{2}(t, \xi(t))+\frac{\gamma}{\alpha^{4}} u(t, \xi(t)) \\
& -\frac{1}{\alpha^{2}} p *\left(h(u)+\frac{\alpha^{2}}{2} u_{x}^{2}-\frac{1}{2} u^{2}+\frac{\gamma}{\alpha^{2}} u\right) .
\end{aligned}
$$

For $x=\xi(t)$, since $u_{x x}(t, \xi(t))=0$, we arrive at

$$
m^{\prime}(t) \leq-\frac{1}{2} m^{2}(t)+K(u) \quad \text { a.e. on }(0, T)
$$

where

$$
K(u)=\frac{\gamma}{\alpha^{4}}\|u\|_{L^{\infty}}+\frac{1}{\alpha^{2}}\left(\sup _{|v| \leq\|u\|_{L^{\infty}}}|h(v)|\right)+\left\|\frac{1}{\alpha^{2}} p *\left(h(u)+\frac{1}{2} u^{2}+\frac{\gamma}{\alpha^{2}} u\right)\right\|_{L^{\infty}} .
$$

Note that

$$
\|u\|_{L^{\infty}}^{2} \leq \frac{1}{2}\|u\|_{1}^{2} \leq \max \left(1, \frac{1}{\alpha^{2}}\right) E\left(u_{0}\right) \leq \max \left(1, \frac{1}{\alpha^{4}}\right)\left\|u_{0}\right\|_{1}^{2} .
$$


By Young's inequality, we get

$$
\begin{aligned}
& \|p * h(u)\|_{L^{\infty}} \leq \sup _{|v| \leq \max \left(1, \frac{1}{\alpha^{2}}\right)\left\|u_{0}\right\|_{1}}|h(v)|, \\
& \left\|p * u^{2}\right\|_{L^{\infty}} \leq \max \left(1, \frac{1}{\alpha^{4}}\right)\left\|u_{0}\right\|_{1}^{2}
\end{aligned}
$$

and

$$
\|p * u\|_{L^{\infty}} \leq \max \left(1, \frac{1}{\alpha^{2}}\right)\left\|u_{0}\right\|_{1}
$$

So, it follows that

$$
m^{\prime}(t) \leq-\frac{1}{2} m^{2}(t)+K_{0}
$$

where (note that $A_{0}^{2}=K_{0}$ )

$$
\begin{aligned}
& G_{0}=\sup _{|v| \leq \max \left(1, \frac{1}{\alpha^{2}}\right)\left\|u_{0}\right\|_{1}}|h(v)|, \\
& K_{0}=\frac{2 \gamma}{\alpha^{4}} \max \left(1, \frac{1}{\alpha^{2}}\right)\left\|u_{0}\right\|_{1}+\frac{1}{2 \alpha^{2}} \max \left(1, \frac{1}{\alpha^{4}}\right)\left\|u_{0}\right\|_{1}^{2}+\frac{2}{\alpha^{2}} G_{0} .
\end{aligned}
$$

The absolute continuity of the locally Lipschitz function $m(t)$ allows us to perform an integration over $[0, t]$ and to have

$$
m(t) \leq m(0)-\frac{1}{2} \int_{0}^{t} m^{2}(\tau) d \tau+K_{0} t, \quad t \in[0, T)
$$

We claim now that $m(t)<-c$ for all $t \in(0, T)$, where $c>\sqrt{2 K_{0}}$ is fixed arbitrarily provided that $m(0)<-c$. In fact, assuming the contrary, in view of $m(t)$ being continuous, ensure the existence of $t_{0} \in(0, T)$ such that $m(t)<-c$ in $\left(0, t_{0}\right)$ and $m\left(t_{0}\right)=c$. Then we deduce that

$$
m(t) \leq m(0)-\int_{0}^{t} K_{0} d \tau+K_{0} t=m(0)<-c, \quad t \in\left[0, t_{0}\right]
$$

and a contradiction appears as we take $t=t_{0}$. Using (11), we get

$$
\begin{aligned}
m^{\prime}(t) & \leq-\frac{1}{2} m^{2}(t)+K_{0} \leq-\frac{1}{2} m^{2}(t)+\left(\frac{1}{2}-\varepsilon\right) c^{2} \\
& \leq-\frac{1}{2} m^{2}(t)+\left(\frac{1}{2}-\varepsilon\right) m^{2}(t) \\
& \leq-\varepsilon m^{2}(t) \quad \text { a.e. on }(0, T)
\end{aligned}
$$

where $\varepsilon \in\left(0, \frac{1}{2}-\frac{K_{0}}{c^{2}}\right)$. Since $m(t)<-c$, and $m(t)$ is locally Lipschitz, it follows that $\frac{1}{m(t)}$ is locally Lipschitz as well. This gives

$$
\frac{d}{d t}\left(\frac{1}{m(t)}\right)=-\frac{m^{\prime}(t)}{m^{2}(t)} \geq \varepsilon \quad \text { a.e. on }(0, T) .
$$


Integration of this inequality yields

$$
-\frac{1}{m(t)}+\frac{1}{m(0)} \leq-\varepsilon t
$$

Since $m(t)<0$, we obtain

$$
0 \leq t<\frac{1}{\varepsilon(-m(0))}, \quad t \in[0, T) .
$$

In fact, as a consequence of these considerations, we obtain that the maximal existence time

$$
T \leq \frac{1}{\varepsilon(-m(0))} \quad \text { for all } \varepsilon \in\left(0, \frac{1}{2}-\frac{K_{0}}{c^{2}}\right) .
$$

An estimation from the above for $T$ is obtained immediately, namely

$$
T \leq \frac{2 c^{2}}{(-m(0))\left(c^{2}-2 K_{0}\right)}
$$

The conclusion is reached by letting $c \rightarrow-m(0)$.

\section{Stability of solitary waves}

In this section, we discuss the stability of solitary wave solutions of Eq. (4) with $h(u)=$ $2 \omega u+\frac{p+2}{2} u^{p+1}$ and $g(u)=u^{p}$. In this case, Eq. (4) turns into the following equation:

$$
\left\{\begin{array}{l}
u_{t}-\alpha^{2} u_{x x t}+\left(2 \omega u+\frac{p+2}{2} u^{p+1}\right)_{x}+\gamma u_{x x x}=\alpha^{2}\left(\frac{p}{2} u^{p-1} u_{x}^{2}+u^{p} u_{x x}\right)_{x} \\
u(0, x)=u_{0}(x)
\end{array}\right.
$$

where $p>0$. When $h(u)=2 \omega u+\frac{p+2}{2} u^{p+1}$ and $g(u)=u^{p}$, the conservation law $F(u)$ takes the form

$$
F(u)=\int_{R}\left(\omega u^{2}+\frac{u^{p+2}}{2}+\frac{\alpha^{2} u^{p}}{2} u_{x}^{2}-\frac{\gamma}{2} u_{x}^{2}\right) d x .
$$

The appropriate notion of stability for the solitary waves here is orbital stability: a wave starting close to a solitary wave should stay close, as long as it exists, to some translate of the solitary wave. The orbit of a solitary wave is the set of all its translates [17].

We define the orbit $\varphi(\cdot-\eta)=\{\tau(\eta) \varphi(\cdot): \eta \in R\}$, where $\tau$ is a one-parameter group of unitary operators on $H^{2}$ defined by $\tau(s) u(\cdot)=u(\cdot-s), s \in R, u \in H^{2}$. They may be interpreted physically as 'solitary waves' or 'bound states'.

Definition The $\varphi$-orbit is stable if for all $\varepsilon>0$, there exists $\delta>0$ with the following property. If $\|u(0, \cdot)-\varphi(\cdot)\|_{H^{2}}<\delta$ and $u$ is a solution of Eq. (12) in some interval $[0, T)$ with $u(0, \cdot)=u_{0}$, then $u$ can be continued to a solution in $0 \leq t<\infty$ and

$$
\sup _{t} \inf _{\eta}\|u(t, \cdot)-\varphi(\cdot-\eta)\|_{H^{2}}<\varepsilon
$$

Otherwise, the $\varphi$-orbit is called unstable. 
Definition can be seen in reference [13].

Substituting $u(x, t)=\varphi(x-c t)=\varphi(\sigma)$ into Eq. (12) and integrating once with respect to $\sigma$, we obtain

$$
c \varphi-c \alpha^{2} \varphi^{\prime \prime}-2 \omega \varphi-\frac{p+2}{2} \varphi^{p+1}-\gamma \varphi^{\prime \prime}+\alpha^{2}\left(\frac{p}{2} \varphi^{p-1} \varphi^{2}+\varphi^{p} \varphi^{\prime \prime}\right)=0 .
$$

In terms of the functionals $E$ and $F$,

$$
\begin{aligned}
& E^{\prime}(\varphi)=\varphi-\alpha^{2} \varphi^{\prime \prime}, \\
& F^{\prime}(\varphi)=2 \omega \varphi+\frac{p+2}{2} \varphi^{p+1}+\gamma \varphi^{\prime \prime}-\alpha^{2}\left(\frac{p}{2} \varphi^{p-1} \varphi^{\prime 2}+\varphi^{p} \varphi^{\prime \prime}\right),
\end{aligned}
$$

and according to Eq. (13), we can obtain that $c E^{\prime}(\varphi)-F^{\prime}(\varphi)=0$, where $E^{\prime}$ and $F^{\prime}$ are the Fréchet derivatives of $E$ and $F$, respectively. To study the orbital stability of the solitary waves of Eq. (12), we need the operator $H_{c}$ and the function $d(c)=c E(\varphi)-F(\varphi)$. The linearized operator $H_{c}$ around $\varphi$ is defined by

$$
\begin{aligned}
H_{c}= & c E^{\prime \prime}(\varphi)-F^{\prime \prime}(\varphi) \\
= & \alpha^{2} \partial_{x}\left[\left(\varphi^{p}-\left(c+\frac{\gamma}{\alpha^{2}}\right)\right) \partial_{x}\right]+\alpha^{2}\left(p \varphi^{p-1} \varphi^{\prime \prime}+\frac{p(p-1)}{2} \varphi^{p-2} \varphi^{\prime 2}\right) \\
& -\frac{(p+2)(p+1)}{2} \varphi^{p}+c-2 \omega .
\end{aligned}
$$

Lemma 4.1 For each $c \in(2 \omega, \infty), H_{c}=c E^{\prime \prime}(\varphi)-F^{\prime \prime}(\varphi)$ has a unique simple negative eigenvalue, zero is a simple eigenvalue, and the rest of its spectrum is bounded away from zero.

Proof For any $u, v \in H^{2}$, we have $\int_{R} u_{x x} v d x=\int_{R} u v_{x x} d x$ and

$$
\int_{R} \varphi^{p} u_{x x} v d x=\int_{R} p \varphi^{p-1} \varphi_{x} v_{x} u d x+\int_{R} \varphi^{p} v_{x x} u d x-\int_{R} p \varphi^{p-1} \varphi_{x} u_{x} v d x
$$

Therefore,

$$
\left\langle\partial_{x}\left(\varphi^{p}-\left(c+\frac{\gamma}{\alpha^{2}}\right)\right) \partial_{x} u, v\right\rangle=\left\langle\partial_{x}\left(\varphi^{p}-\left(c+\frac{\gamma}{\alpha^{2}}\right)\right) \partial_{x} v, u\right\rangle .
$$

By (14), we know that $H_{c}$ is a self-adjoint operator and

$$
\begin{aligned}
H_{c} \varphi^{\prime}= & \alpha^{2}\left[\left(\varphi^{p}-\left(c+\frac{\gamma}{\alpha^{2}}\right)\right) \varphi^{\prime \prime \prime}\right]+\alpha^{2}\left(2 p \varphi^{p-1} \varphi^{\prime} \varphi^{\prime \prime}+\frac{p(p-1)}{2} \varphi^{p-2} \varphi^{\prime 3}\right) \\
& -\frac{(p+2)(p+1)}{2} \varphi^{p} \varphi^{\prime}+(c-2 \omega) \varphi^{\prime} .
\end{aligned}
$$

Differentiating (13) with respect to $\sigma$, we find that the right-hand side of Eq. (15) equals zero, that is, $H_{c} \varphi^{\prime}=0$. The behavior of the function $\varphi$ tells us that $\varphi^{\prime}$ has exactly one zero. So, the zero eigenvalue of the operator $H_{c}$ is simple, and by using the Sturm-Liouville theorem, we have that $H_{c}$ only has a negative eigenvalue. Using Weyl's theorem, the essential spectrum of $H_{c}$ belongs to $\left[\frac{c-2 \omega}{\alpha^{2}},+\infty\right)$. That completes the proof. 
As for the above results, it is known [13] that stability would be ensured by the convexity of the scalar function $d(c)=c E(\varphi)-F(\varphi), c>2 \omega$. So, we obtain the following theorem under the condition $2 \omega \alpha^{2}+\gamma=0$.

Theorem 4.1 Suppose that $\varphi(\cdot-\eta)$ is a solitary wave solution for $c>2 \omega$, if $d^{\prime \prime}(c)>0$, then $\varphi(\cdot-\eta)$ is stable.

Define the functionals

$$
I(u)=I(u ; \omega, c)=\int_{R}\left[(c-2 \omega) u^{2}+\alpha^{2}(c-2 \omega) u_{x}^{2}\right] d x
$$

and

$$
K(u)=\int_{R}\left(u^{p+2}+\alpha^{2} u^{p} u_{x}^{2}\right) d x .
$$

For $\lambda>0$, we consider the following constrained minimization problem on $H^{2}$ :

$$
M_{\lambda}=\left\{\inf I(u): u \in H^{2}, K(u)=\lambda\right\} .
$$

Then if $\psi \in H^{2}$ achieves the minimum of problem (16), for some $\lambda>0$, then there exists a Lagrange multiplier $\vartheta$ such that

$$
(2 c-4 \omega) \psi-\alpha^{2}(2 c-4 \omega) \psi^{\prime \prime}=\vartheta\left[(p+2) \psi^{p+1}-\alpha^{2}\left(p \psi^{p-1} \varphi^{2}+2 \varphi^{p} \varphi^{\prime \prime}\right)\right] .
$$

Hence $\varphi=\vartheta^{\frac{1}{p}} \psi$ is a solution of solitary wave equation (13). By homogeneity of $I(u)$ and $K(u), \varphi$ satisfies

$$
m=m(\omega, c)=\inf \left\{\frac{I(u)}{K(u)^{\frac{2}{p+2}}}: u \in H^{2}, K(u)>0\right\},
$$

and it follows that

$$
M_{\lambda}=m \lambda^{\frac{2}{p+2}}
$$

Multiplying solitary wave equation (13) by $\varphi$ and integrating the resulting equation gives $I(\varphi)=\frac{(p+2)}{2} K(\varphi)$.

We say that $\psi_{k}$ is a minimizing sequence if for some $\lambda>0, \lim _{k \rightarrow \infty} I\left(\psi_{k}\right)=M_{\lambda}$ and $\lim _{k \rightarrow \infty} K\left(\psi_{k}\right)=\lambda$.

Theorem 4.2 Let $\left\{\psi_{k}\right\}$ be a minimizing sequence for some $\lambda>0$. If $c>2 \omega$, then there exists a subsequence $\left\{\psi_{k_{j}}\right\}$, scalars $y_{j} \in R$ and $\psi \in H^{2}$ such that $\psi_{k_{j}} \rightarrow \psi$ in $H^{2}$. The function $\psi$ achieves the minimum $I(\psi)=M_{\lambda}$ subject to the constraint $K(\psi)=\lambda$.

Proof The result is an application of the concentration compactness lemma of Lions [19, 20]. Proof is similar to [21-23] according to the definition of $I(u)$ and $K(u)$.

In this section, we show that the stability of solitary wave solutions is determined by the convexity of the function $d(c)$. 
We state the basic properties of the function $d$.

A simple calculation gives

$$
d(c)=c E(u)-F(u)=\frac{1}{2}(I(u)-K(u)) .
$$

By using $I(\varphi)=\frac{(p+2)}{2} K(\varphi)$, we obtain

$$
d(c)=\frac{p}{2(p+2)} I(\varphi)=\frac{p}{4} K(\varphi)=\frac{p}{4}\left(\frac{2}{p+2} m\right)^{\frac{p+2}{p}} .
$$

Therefore, $d$ is well defined, and we may deduce its properties by examining the function $m(\omega, c)$.

Lemma 4.2 For fixed $\omega \in R, m(\omega, c)$ is monotonically increasing in $c$.

Proof We assume that $\varphi_{c_{1}}, \varphi_{c_{2}}$ are solutions of Eq. (13) corresponding to $c=c_{1}, c=c_{2}$, respectively. Without loss of generality, let be $c_{1}<c_{2}$, then we have

$$
\begin{aligned}
& m\left(\omega, c_{1}\right) \leq \frac{I_{c}\left(\varphi_{c_{2}} ; \omega, c_{1}\right)}{K\left(\varphi_{c_{2}}\right)^{\frac{2}{p+2}}}=\frac{\int_{R}\left[\left(c_{1}-2 \omega\right) \varphi_{c_{2}}^{2}+\alpha^{2}\left(c_{1}-2 \omega\right)\left(\varphi_{c_{2}}^{\prime}\right)^{2}\right] d x}{K\left(\varphi_{c_{2}}\right)^{\frac{2}{p+2}}} \\
& =\frac{\int_{R}\left[\left(c_{2}-2 \omega\right) \varphi_{c_{2}}^{2}+\alpha^{2}\left(c_{2}-2 \omega\right)\left(\varphi_{c_{2}}^{\prime}\right)^{2}\right] d x}{K\left(\varphi_{c_{2}}\right)^{\frac{2}{p+2}}} \\
& +\frac{-c_{2} \int_{R}\left[\varphi_{c_{2}}^{2}+\alpha^{2}\left(\varphi_{c_{2}}^{\prime}\right)^{2}\right] d x+c_{1} \int_{R}\left[\varphi_{c_{2}}^{2}+\alpha^{2}\left(\varphi_{c_{2}}^{\prime}\right)^{2}\right] d x}{K\left(\varphi_{c_{2}}\right)^{\frac{2}{p+2}}} \\
& =m\left(\omega, c_{2}\right)+\left(c_{1}-c_{2}\right) \frac{\int_{R}\left[\varphi_{c_{2}}^{2}+\alpha^{2}\left(\varphi_{c_{2}}^{\prime}\right)^{2}\right] d x}{K\left(\varphi_{c_{2}}\right)^{\frac{2}{p+2}}} \\
& \leq m\left(\omega, c_{2}\right) .
\end{aligned}
$$

This shows that $m$ is monotonically increasing in $c$, so that by (18), $d$ must be monotonically increasing as well.

A tubular neighborhood around the orbital $\varphi(\cdot-\eta)$ is defined by

$$
U_{\omega, c ; \varepsilon}=\left\{u \in H^{2}: \inf _{\eta \in R}\|u-\varphi(\cdot-\eta)\|_{H^{2}}<\varepsilon\right\} .
$$

It follows from (18) and the fact that $d(c)$ is monotonically increasing in $c$ that

$$
c(u)=d^{-1}\left(\frac{p}{4} K(u)\right) .
$$

The following lemma is helpful in order to prove the stability of solitary waves.

Lemma 4.3 If $d^{\prime \prime}(c)>0$, then there exists $\varepsilon>0$ such that for any $u \in U_{\omega, c ; \varepsilon}$, we have

$$
c(u)[E(u)-E(\varphi)]-[F(u)-F(\varphi)] \geq \frac{1}{4} d^{\prime \prime}(c)|c(u)-c|^{2} .
$$


Proof By using $d^{\prime}(c)=E(\varphi)$ and Taylor's formula, we have the expansion

$$
d(\widetilde{c})=d(c)+E(\varphi)(\widetilde{c}-c)+\frac{1}{2} d^{\prime \prime}(c)(\widetilde{c}-c)^{2}+o\left(|\widetilde{c}-c|^{2}\right)
$$

for $\tilde{c}$ near $c$. Using the continuity of $c(u)$ and choosing $\varepsilon$ sufficiently small, we get that

$$
\begin{aligned}
d(c(u)) & \geq d(c)+E(\varphi)(c(u)-c)+\frac{1}{4} d^{\prime \prime}(c)(c(u)-c)^{2} \\
& =c(u) E(\varphi)-F(\varphi)+\frac{1}{4} d^{\prime \prime}(c)(c(u)-c)^{2} .
\end{aligned}
$$

It follows from (18) and (19) that $K\left(\varphi_{c(u)}\right)=\frac{4}{p} d(c)=K(u)$. Since $\varphi_{c(u)}$ is a minimizer of $I(u ; \omega, c(u))$ subject to the constraint $K(u)=K\left(\varphi_{c(u)}\right)$, we then have

$$
\begin{aligned}
c(u) E(u)-F(u) & =\frac{1}{2}(I(u ; \omega, c(u))-K(u)) \\
& \geq \frac{1}{2}\left(I\left(\varphi_{c(u)} ; \omega, c(u)\right)-K\left(\varphi_{c(u)}\right)\right)=d(c(u))
\end{aligned}
$$

and

$$
c(u) E(u)-F(u) \geq c(u) E(\varphi)-F(\varphi)+\frac{1}{4} d^{\prime \prime}(c)(c(u)-c)^{2} .
$$

Proof of Theorem 4.1 Suppose that $\varphi(\cdot-\eta)$ is unstable. Then there exists $\varepsilon_{0}$ and a sequence of initial data $u_{k}(0)$ satisfying

$$
\inf _{\eta}\left\|u_{k}(0)-\varphi(\cdot)\right\|_{H^{2}} \rightarrow 0
$$

however,

$$
\operatorname{supinf}_{\eta}\left\|u_{k}(t)-\varphi(\cdot-\eta)\right\|_{H^{2}} \geq \varepsilon_{0}
$$

where $u_{k}(t)$ is a solution of Eq. (12) with initial datum $u_{k}(0)$. Then, by Theorem 2.1, $u_{k}$ is continuous in $t$, and there exist times $t_{k}$ such that

$$
\inf _{\eta}\left\|u_{k}\left(t_{k}\right)-\varphi(\cdot-\eta)\right\|_{H^{2}}=\varepsilon_{0}
$$

When $t_{k} \rightarrow 0,\left\|u_{k}(0)-\varphi(\cdot)\right\|_{H^{2}} \rightarrow 0, E$ and $F$ are invariants of (12) so that

$$
E\left(u_{k}\left(t_{k}\right)\right)=E\left(u_{k}(0)\right) \rightarrow E(\varphi), \quad F\left(u_{k}\left(t_{k}\right)\right)=F\left(u_{k}(0)\right) \rightarrow F(\varphi),
$$

by Lemma 4.3 , we have

$$
\begin{aligned}
& c\left(u_{k}\left(t_{k}\right)\right)\left[E\left(u_{k}\left(t_{k}\right)\right)-E(\varphi)\right]-\left[F\left(u_{k}\left(t_{k}\right)\right)-F(\varphi)\right] \\
& \quad \geq \frac{1}{4} d^{\prime \prime}(c)\left|c\left(u_{k}\left(t_{k}\right)\right)-c\right|^{2} .
\end{aligned}
$$

This implies that $c\left(u_{k}\left(t_{k}\right)\right) \rightarrow c$, as $k \rightarrow \infty$, since $u_{k}\left(t_{k}\right)$ is uniformly bounded for $k$. 
The continuity of $d$ implies that

$$
\lim _{k \rightarrow \infty} K\left(u_{k}\left(t_{k}\right)\right)=\lim _{k \rightarrow \infty}\left(\frac{4}{p} d\left(c\left(u_{k}\left(t_{k}\right)\right)\right)\right)=\frac{4}{p} d(c) .
$$

Using (17) and the fact that $d(c)=c E(\varphi)-F(\varphi)$, we have

$$
\begin{aligned}
\frac{1}{2} I\left(u_{k}\left(t_{k}\right)\right)= & c E\left(u_{k}\left(t_{k}\right)\right)-F\left(u_{k}\left(t_{k}\right)\right)+\frac{1}{2} K\left(u_{k}\left(t_{k}\right)\right) \\
= & d(c)-F\left(u_{k}\left(t_{k}\right)\right)+F(\varphi)-c\left(E(\varphi)-E\left(u_{k}\left(t_{k}\right)\right)\right) \\
& +\frac{1}{2} K\left(u_{k}\left(t_{k}\right)\right),
\end{aligned}
$$

so it follows from (21) and (22) that

$$
\lim _{k \rightarrow \infty} I\left(u_{k}\left(t_{k}\right)\right)=\frac{2(p+2)}{p} d(c) .
$$

From (22) and (23), $u_{k}\left(t_{k}\right)$ is a minimizing sequence for the pair $I, K$ and thus by Theorem 4.2 has a subsequence, named $u_{k_{j}}\left(t_{k_{j}}\right)$, that converges in $H^{2}$ to some $\varphi$. This contradicts (20). The proof is completed.

\section{Competing interests}

The authors declare that they have no competing interests.

\section{Authors' contributions}

All authors typed, read and approved the final manuscript.

\section{Acknowledgements}

This work was supported by Grant No. 13-FF-46 of the Dicle University of Scientific Research Projects Coordination (DUBAP), Diyarbakir, Turkey.

\section{Received: 21 May 2013 Accepted: 3 September 2013 Published: 07 Nov 2013}

\section{References}

1. Dullin, HR, Gottwald, GA, Holm, DD: An integrable shallow water equation with linear and nonlinear dispersion. Phys. Rev. Lett. 87, 1945-1948 (2001)

2. Johnson, RS: Camassa-Holm, Korteweg-de Vries and related models for water waves. J. Fluid Mech. 455, 63-82 (2002)

3. Tian, L, Gui, G, Liu, Y: On the Cauchy problem and the scattering problem for the Dullin-Gottwald-Holm equation. Commun. Math. Phys. 257, 667-701 (2005)

4. Hakkaev, S: Stability of peakons for an integrable shallow water equation. Phys. Lett. A 354, 137-144 (2006)

5. Constantin, A, Strauss, WA: Stability of the Camassa-Holm solitons. J. Nonlinear Sci. 12, 415-422 (2002)

6. Yin, Z: Well-posedness, blow up, and global existence for an integrable shallow water equation. Discrete Contin. Dyn. Syst. 11, 393-411 (2004)

7. Yin, Z: Global existence and blow-up for a periodic integrable shallow water equation with linear and nonlinear dispersion. Dyn. Contin. Discrete Impuls. Syst. Ser. A Math. Anal. 12, 87-101 (2005)

8. Zhang, S, Yin, Z: On the blow-up phenomena of the periodic Dullin Gottwald Holm equation. J. Math. Phys. 49, 113504 (2008)

9. Zhou, Y: Blow-up of solutions to the DGH equation. J. Funct. Anal. 250, 227-248 (2007)

10. Lu, D, Peng, D, Tian, L: On the well-posedness problem for the generalized Dullin-Gottwald-Holm equation. Int. J. Nonlinear Sci. 1, 178-186 (2006)

11. Naz, R, Naeem, I, Abelman, S: Conservation laws for Camassa-Holm equation, Dullin-Gottwald-Holm equation and generalized Dullin-Gottwald-Holm equation. Nonlinear Anal., Real World Appl. 10, 3466-3471 (2009)

12. Liu, X, Yin, Z: Local well-posedness and stability of peakons for a generalized Dullin-Gottwald-Holm equation. Nonlinear Anal. 74, 2497-2507 (2011)

13. Grillakis, M, Shatah, J, Strauss, W: Stability theory of solitary waves in the presence of symmetry I. J. Funct. Anal. 74 160-197 (1987)

14. Kato, T, Ponce, G: Commutator estimation and the Euler and Navier Stokes Equation. Commun. Pure Appl. Math. 41 891-907 (1998)

15. Constantin, A, Molinet, L: The initial value problem for a generalized Boussinesq equation. Differ. Integral Equ. 15 1061-1072 (2002) 
16. Constantin, A, Escher, J: Wave breaking for nonlinear nonlocal shallow water equation. Acta Math. 181, 229-243 (1998)

17. Constantin, A, Strauss, WA: Stability of a class of solitary waves in compressible elastic rods. Phys. Lett. A 270, 140-148 (2000)

18. Wu, X, Yin, Z: Well-posedness and blow-up phenomena for the generalized Degasperis-Procesi equation. Nonlinear Anal. 73, 136-146 (2010)

19. Lions, PL: The concentration compactness principle in the calculus of variations. The locally compact case, Part 1 . Ann. Inst. Henri Poincaré, Anal. Non Linéaire 1, 109-145 (1984)

20. Lions, PL: The concentration compactness principle in the calculus of variations. The locally compact case, Part 2. Ann. Inst. Henri Poincaré, Anal. Non Linéaire 1, 223-283 (1984)

21. Albert, JP: Concentration compactness and the stability of solitary wave solutions to nonlocal equations. Contemp. Math. 221, 1-29 (1999)

22. Levandosky, SP, Liu, Y: Stability of solitary waves of a generalized Ostrovsky equation. SIAM J. Math. Anal. 38, 985-1011 (2006)

23. Liu, Y, Varlamov, V: Stability of solitary waves and weak rotation limit for the Ostrovsky equation. J. Differ. Equ. 203, 159-183 (2004)

10.1186/1687-2770-2013-226

Cite this article as: Dündar and Polat: Blow-up phenomena and stability of solitary waves for a generalized

Dullin-Gottwald-Holm equation. Boundary Value Problems 2013, 2013:226

\section{Submit your manuscript to a SpringerOpen ${ }^{\circ}$ journal and benefit from:}

- Convenient online submission

- Rigorous peer review

- Immediate publication on acceptance

- Open access: articles freely available online

- High visibility within the field

- Retaining the copyright to your article 